\title{
Aprendizaje basado en proyectos y learn by doing como herramientas de independencia y autonomía en el desarrollo de la creatividad de la asignatura "Dirección de actores"
}

\section{Project-based learning and learn by doing as tools of independence and autonomy in the development of creativity in the subject "Directing actors"}

\section{Saida Santana Mahmut}

Fecha de recepción: 13/02/2021; Fecha de revisión: 22/04/2021 Fecha de aceptación: 05/10/2021

Cómo citar este artículo:

Santana, S. (2021) Aprendizaje basado en proyectos y learn by doing como herramientas de independencia y autonomía en el desarrollo de la creatividad de la asignatura "Dirección de actores". Revista de Innovación y Buenas Prácticas Docentes, 10(2), 13-26.

Autor de Correspondencia: ssantana@nebrija.es

\section{Resumen:}

Enseñar creatividad en materias prácticas es un reto para docente y alumno. La docencia de la creatividad ha de incluir metodologías de aprendizaje que contemplen el protagonismo del alumnado en su propio proceso de aprendizaje. Creatividad y autonomía van de la mano en materias en las que el alumno debe adquirir herramientas de liderazgo e independencia para el desempeño de su futura profesión. Este artículo recoge la sistematización de una metodología de Aprendizaje Basado en Proyectos -ABP- y learn by doing usada durante siete años en la asignatura de "Dirección de actores" del Grado de Comunicación audiovisual y el Máster de realización de series de ficción de la Universidad Antonio de Nebrija, para fomentar la creatividad, y permitir la adquisición gradual de autonomía e independencia del discente. Se explica el sistema de trabajo empleado para la adquisición de habilidades, conocimientos y aptitudes del alumnodirector de forma gradual y por fases a través de talleres prácticos. En la fase 1 se imparten protocolos y conocimientos técnicos, en la fase 2 se imparte taller 1 , en la fase 3, taller 2 y en la fase 4 , el alumno trabaja de modo autónomo y práctico la dirección y grabación de un monólogo.

Palabras clave: Aprendizaje Basado en Proyectos, learn by doing, dirección de actores, creatividad

\begin{abstract}
:
Teaching creativity in practical subjects is a challenge for both teacher and student. Teaching creativity must include learning methodologies that consider the role of the student in their own learning process. Creativity and autonomy go hand in hand in subjects in which the student must acquire leadership and independence tools to carry out their future profession. This article collects the systematization of a Project-Based Learning methodology -ABP- and learn by doing used for seven years in "Directing actors" subject of the Degree in Audiovisual Communication and the fiction series Realization Master's Degree in Antonio de Nebrija University, to promote creativity, and allow the gradual acquisition of autonomy and independence of the student. This article contains the explanation of the work system for the gradual and phased acquisition of skills, knowledge and aptitudes of the student.director, which allows the passage from knowledge to action, through practical workshops. In phase 1 protocols and technical knowledge are taught, in phase 2 Workshop 1 is taught, in phase 3, Workshop 2 and in phase 4 , the student works autonomously and practically directing and recording a monologue for their final exam.
\end{abstract}

Key Words: Project-Based Learning, learn by doing, directing actors, creativity. 


\section{INTRODUCCIÓN}

Antes de adentrarnos en la descripción de la asignatura "Dirección de actores" es importante mencionar que quien suscribe la ha impartido en Grado los años: 2011-2012, 2012-2013, 2014-2015, 2015-2016, 2016-2017, 2017-2018; y en Máster los años: 20152016, 2016-2017, 2017-2018, 2018-2019, 2019-2020, lo que supone unos 75 alumnos de Máster y unos 150 alumnos de Grado. La única diferencia entre Grado y Máster está en el número de créditos. En Grado la asignatura tiene 6 y en Máster 4 pero, tanto la metodología usada como las Actividades dirigidas solicitadas -talleres- y el trabajo final, son iguales para ambas formaciones. La única diferencia está en que en Máster ambos talleres se hacen de manera grupal para agilizar, dada la merma de horas, y en Grado se desarrollan de manera individual. En el Máster desaparece la grabación de monólogo como examen final. La decisión de hacerlo así se debe a que los alumnos del Máster de realización de series de ficción en un futuro trabajarán codirigiendo la mayoría de las veces. Practicar conjuntamente con otros directores permitía dar el mismo contenido en menor tiempo, a la vez que introducíamos la competencia de trabajo en equipo, que les será necesaria el día de mañana.

Durante mucho tiempo la dirección de actores no ha sido considerada como parte fundamental de la complejidad de la dirección. Los directores debían ser buenos en los aspectos técnicos de realización, pero dejaban al azar o a la espontaneidad del momento sus directrices al actor. Esto ha cambiado considerablemente en los últimos años, en los que se ha entendido la importancia del trabajo del director con el actor. Ahora bien, ¿hay una metodología concreta?, ¿acaso el actor sigue un único sistema en el vasto universo de la construcción del personaje? Si leemos a teóricos de la dirección escénica como Meyerhold, Nemiróvich-Dánchenko o Grotowski, o incluso si retrocedemos en el tiempo hasta el duque de Saxe-Meininger-considerado el primer director de actores- podremos ver puntos en común, pero ninguno ofrece un método sistematizado que pueda seguir un director para triunfar en esta ardua tarea. Siendo consciente de la dificultad de enseñar a dirigir actores y de los miles de caminos que se podrían recorrer, el objetivo principal de la asignatura es marcar un esquema básico de trabajo, que más adelante, en su entrenamiento profesional, el alumno-director pueda seguir enriqueciendo.

Dentro del abanico de facetas de la dirección, la dirección de actores es sin duda de las más creativas. En realidad, es doblemente creativa pues la creatividad del director fomenta la del actor. La pregunta de partida a la hora de diseñar la guía docente y el contenido fue: ¿cómo enseñar creatividad para dirigir la creatividad del actor? Para lograrlo fue de gran ayuda la formación académica y la experiencia profesional de quien suscribe este artículo, como actriz, directora, docente y coach personal y actoral.

Aunque suene paradójico, la estructuración, la sistematización de pautas y esquemas de trabajo son las que crean una base sobre la que se puede crear. Si no se acota no hay posibilidad.

La creatividad es una habilidad que permite que surjan ideas nuevas o que se perfeccionen otras o ayuda a solucionar problemas (Sternberg, 1999). La creatividad puede enmarcarse en tres perspectivas: la producción creativa, el pensamiento divergente 0 rasgo de la personalidad (Barron \& Harrington,1981). En este trabajo abordamos la creatividad del director en cuanto a la producción creativa, al producto concreto de la escena que dirige y el resultado actoral; $y$ al pensamiento divergente que pone en funcionamiento para desatar el mecanismo creativo. 
Los alumnos que llegan a la asignatura, tanto en el Máster como en el Grado, llegan faltos de creatividad y no porque no sean creativos. Al contrario, habitualmente esto ocurre porque tienen respeto a la materia, ya que dicen "no tener idea de cómo dirigir a los actores y dirigirse a ellos. Confiesan no saber por dónde empezar y, sobre todo, consideran que es una tarea subjetiva. Además, en la mayoría de los casos, descubrir que han de hacer funciones de actor en el proceso les incomoda. Esas resistencias son el mayor freno a su creatividad. El diseño inicial de la asignatura tenía que tener entonces como objetivo principal encontrar el modo de objetivizar y tangibilizar lo que ellos veían como intangible y subjetivo. Con los años hemos comprobado reiteradamente que ofrecerles un método de trabajo, una sistematización de la tarea de dirección de actores, es la única manera de lograrlo y hacer que saquen a la luz su creatividad como directores y superen sus resistencias. Si no saben lo que tienen que hacer, será más difícil que se arriesguen. Si no tienen unas pautas que seguir no podrá brotar su creatividad, no podrán crear y, en consecuencia, no podrán ser autónomos e independientes.

La dirección de actores, al ser una parte más del complejo trabajo del director, entremezcla lo artístico y lo técnico. Por ello la impartición de esta asignatura contempla ambas visiones. Además, la metodología de trabajo por fases permite al alumno ir adquiriendo confianza, autonomía y liderazgo gradualmente. El aprendizaje basado en proyectos -ABP- y learn by doing son las piezas claves en el desarrollo de los dos talleres de "Dirección de actores" que tiene la asignatura y el examen final 100\% práctico y profesional: dirección y grabación de un monólogo.

\section{DESARROLLO DE LA EXPERIENCIA DE INNOVACIÓN}

\subsection{Hipótesis y objetivos}

Las hipótesis de partida de la asignatura y del trabajo en sí son:

La propuesta de talleres prácticos como actividades de aprendizaje basado en proyecto y learn by doing son fuente de creatividad y autonomía.

Cuanta más estructura se le ofrece al alumno antes y durante el taller, mayor será su grado de autonomía lo que potenciará más y mejor su creatividad. El objetivo general que persigue este trabajo es mostrar un método sistematizado de trabajo ABP y learn by doing en la asignatura universitaria "Dirección de actores" para fomentar la creatividad y autonomía del futuro director, que pueda servir de guía a otros docentes que se adentren en una materia aparentemente abstracta.

Este objetivo general se concreta en los siguientes objetivos específicos:

1. Explicar el rol docente como instructor, guía y ayudante según la fase del proceso.

2. Explicar el contenido de la asignatura.

3. Exponer la estructura e itinerario formativo de los talleres.

4. Explicar la gradación de contenido y reto en las distintas fases.

5. Exponer la metodología de ABP y learn by doing a través del contenido de la asignatura y resultados. 
6. Mostrar la aplicación de ABP y learn by doing en los resultados de los alumnos.

7. Ofrecer conclusiones del proceso de trabajo.

\subsection{Metodología}

La metodología de este trabajo consiste en una primera fase analítico-descriptiva de contextualización del objeto de estudio, la argumentación de su pertinencia y la creación de un marco teórico de referencia. Dicha fase se aborda con la consulta de fuentes secundarias de libros y artículos relativos a técnicas de interpretación, dirección escénica y de actores, y análisis de guion y escenas y construcción de personaje, así como materiales docentes propios.

En cuanto a los modelos de dirección se han revisado las teorías de NemiróvichDánchenko (2006), Meyerhold (2010) y Grotowski (2009). En lo relativo a las técnicas actorales para elaborar la metodología y el material docente se ha consultado una extensa bibliografía, así como la asistencia de primera mano de la autora a distintos cursos internacionales. Si bien los más significativos para el protocolo han sido: toda la bibliografía del maestro Stanislavski, pues de su Sistema se recogen la mayoría de las herramientas básicas utilizadas. También se han consultado los métodos de Strasberg (2019), Hagen (2012), Adler (2000), Batson (2014), Chejov (1999), Meisner (2003), Boleslavsky (2015), y el compendio de Bartow (2006). El protocolo de las preguntas de Barr (2012) y el de las 13 preguntas del actor al director de Serna (1999) se ha complementado con las nociones elementales de Caine (2003). El trabajo profesional de la autora como directora y actriz interviene en esta fase. Para ello se ha recurrido a la observación directa participante u observación global "en la que el investigador se integra en el medio ambiente del grupo estudiado e interviene en sus prácticas y rituales cotidianos" (De Miguel, 2005, p. 279).

La segunda fase del trabajo consiste en el análisis y exposición de la fase empírica de la asignatura sustentada en Aprendizaje Basado en Proyectos y learn by doing.

La asignatura, a su vez, se divide en una parte analítico-descriptiva y una fase empírica compuesta por dos talleres prácticos y un examen final. La recogida de datos se ha hecho a través de las entregas de los documentos exigidos en los talleres que configuran las Actividades dirigidas de la asignatura.

\subsection{Aprendizaje basado en proyectos y learn by doing.}

El ABP, junto con learn by doing, ha sido la metodología elegida para el trabajo práctico de la asignatura "Dirección de actores", ya que permite que con la explicación de los conocimientos y herramientas explicados en la fase 1, teórica, el alumno pueda trabajar en las propuestas planteadas por el profesor. Para Barrows (1986) ABP es un método de aprendizaje que usa los proyectos como punto de partida para adquirir e integrar nuevos conocimientos. Aunque el docente explique las partes más significativas del temario hasta que el director no dirija a los actores en la escena, no saldrá a la luz la parte más importante de la asignatura, ya que cada actor requerirá una solución diferente. Esto convierte al alumno en el protagonista de su aprendizaje. Además de adquirir competencias como resolución de problemas, comunicación, trabajo en equipo, pensamiento crítico o conciencia de su proceso de aprendizaje, ABP favorece el desarrollo del razonamiento eficaz y la creatividad (Benito \& Cruz, 2005). 
Por su parte, learn by doing es una metodología de aprendizaje constructivista basada en los estudios de Schank (1999). Es otro modelo orientado a la resolución de problemas y aplicación de conocimientos en situaciones reales. Para Schank (1999) los individuos aprenden únicamente haciendo. El alumno es un participante activo en el proceso.

\subsection{Contenido de la asignatura}

Puesto que el aprendizaje y la experiencia es gradual el proceso de trabajo se dividirá en fases. Cada una de ellas va aumentando la creatividad del alumno y su consecuente autonomía. La primera fase de la asignatura, la descriptiva, corresponde a la fase 1 y se divide a su vez en tres bloques fundamentales: el primer bloque referido al conocimiento de las técnicas interpretativas más usadas con foco en Stanislavski, otro referido a las competencias de un buen director, y un tercero enfocado en el análisis y construcción del personaje. El sistema de Stanislavski ha sido el eje vertebral de todo el contenido, pues todas las metodologías de carácter realista posteriores a su Sistema son derivaciones de él. Además, tal y como Melendres afirmó en su momento, "la obra de Stanislavski parece destinada fundamentalmente a la formación de los directores de escenas" (2000, p. 92).

Para que el alumno adquiera las competencias básicas que necesita un director de actores los contenidos recogen lo siguiente: conocimiento de técnicas interpretativas, manejo de competencias genéricas de dirección y manejo de competencias específicas de dirección de actores.

En cuanto al conocimiento de técnicas interpretativas se hace un recorrido por las teorías más significativas y de Stanivslavski se enseñan herramientas interpretativas tales como el sí mágico, memoria emotiva, acciones físicas transformadoras y memoria sensorial. Se explica el trabajo con el objetivo de protagonista y antagonista para generar conflicto. Se busca el superobjetivo del personaje en la obra cinematográfica o serie. Se indaga en la contradicción del personaje y se buscan las circunstancias dadas del personaje en la escena en cuestión. Además, se explican las reglas básicas de la técnica de improvisación y se indica cuándo es apropiado usarla y cuándo no. Esta fase incluye visionado de ejemplos: testimonios y escenas.

El segundo bloque de esta fase 1 trabaja las competencias del director de actores. En este caso la asignatura aborda las características del director eficaz, claves y reglas para dirigir actores, principios del líder, relación con el actor y modelos de dirección de Meyerhold, Nemiróvich-Dánchenko, Stanivslavski, y Grotowski, entre otros. Con todas estas competencias el director hará su DAFO personalizado para descubrir cuáles son sus áreas débiles y pueda mejorarlas en los sucesivos talleres.

En el tercer bloque de esta fase 1 el alumno debe adquirir el manejo de competencias que ayuden al análisis de las escenas y la construcción del personaje. Esta será la guía básica que el director completará para ofrecer al actor. Habitualmente el mayor miedo al que se enfrenta el director es no saber qué decir al actor sobre su personaje, qué pedirle y qué pautas darle. Esta guía ofrece un protocolo que le ayudará a hacer tangible una tarea que les parece abstracta. Se analizará el texto a trabajar buscando subtexto, claves, objetivos, beats, y detalle de acciones. Para la construcción del personaje se trabaja con las 8 preguntas de Barr (2002), más 2 incorporadas por la docente, y las 13 preguntas de Serna (1999). 
Las 8 preguntas de Barr (2002) suponen las preguntas básicas que un actor se hace a la hora de trabajar en la construcción del personaje. Habitualmente la mayor dificultad e incongruencia en la dirección de actores surge porque el director da por hecho que el actor va a construir el personaje de acuerdo a lo que él tiene en su mente. El grave problema es que el guion no da siempre la respuesta a estas preguntas. Para que el actor pueda ser libre y creativo necesita información y el protocolo de la asignatura. Estas 8 preguntas más 2 son el mínimo que un director debe compartir con el actor, para que este no construya un personaje alejado de lo que el director quiere. Para solventar este problema es crucial que el director se adelante y responda profundamente a estas 8 preguntas y se las comunique a cada actor (Tabla 1).

Tabla 1.

Esquema de 8 preguntas de Barr (2002) más 2.

¿Qué clase de persona es usted?

¿Qué más información sobre usted puede deducir de la historia?

¿Ha vivido alguna vez una situación como la que describe la historia?

- Si es así: ¿cuáles eran tus sentimientos?, ¿qué hizo?, ¿cómo reaccionó?

- Si no es así ponga a trabajar su imaginación, uso del sí mágico: ¿qué sentiría si viviera una situación así?

¿Cuál es su situación emocional al principio de la escena?

- ¿De dónde viene?

- ¿Qué estaba haciendo inmediatamente antes del principio de la escena?

- Coreografíe entre 30 y 60 segundos de actividad para implicarse en la realidad de la situación.

Qué es lo que le impulsa durante la escena?

¿Qué es lo que necesita conseguir? (objetivo de Stanislavski)

¿Cuál es el estado emocional durante la escena?

- ¿Cuál es el ritmo de esa emoción?

- ¿Más rápido de lo normal?

- ¿Más lento? ¿más normal?

¿Cuál es el obstáculo u obstáculos? (Conflicto a raíz de un objetivo opuesto del antagonista) Externos

- ¿Quien-es se interponen en tu camino? (estudiar las frases de la otra persona) entender lo que dice y lo que no dice

Internos

- ¿Qué ideas, puntos débiles o experiencias recientes están dificultando la satisfacción de tus necesidades (Jorge Eines lo llama contradicción del personaje)

¿Qué vínculo tiene el personaje con los otros personajes?

¿Qué relación tiene el personaje con el entorno?

Fuente: elaboración propia basada en el esquema del libro de Tony Barr. Actuando para la cámara. Manual de actores para cine y TV. Plot. Madrid, 2002.

A este esquema básico la docente ha añadido 2 preguntas referentes al vínculo con los otros personajes y a la relación con el entorno. Cuanto más específico se sea en estos dos aspectos más credibilidad se conseguirá.

Para complementar el proceso anterior se ofrecen estas 13 preguntas de Serna (1999) del actor al director. Preguntas que necesitan ser respondidas por el director para que el actor pueda ser creativo (Tabla 2).

Tabla 2. 
Esquema de 13 preguntas de Serna (1999).

Vida interior del personaje

Cómo se enfrenta a los conflictos

Su relación con los demás personajes (vínculo)

¿Qué hace cuando está sólo? Es muy importante tener espacio en una película para el personaje en soledad. Será el momento de complicidad del personaje con el público.

Vida personal, profesional y privada

Puntos de vista del personaje

Cambios de actitud del personaje

Personalidad o tono del personaje

¿Qué aprende el actor del personaje? (mensaje)

¿Qué aprende el público de la historia?

Momentos culminantes de revelación del personaje

Información sobre guion

Cómo imagina el director físicamente al personaje. Ejemplos cercanos. Transeúntes, familiares, personajes similares interpretados en el cine o en teatro.

Fuente: Elaboración propia basada en el libro de Serna (1999).

De las 13 preguntas, las referentes a: vida personal, profesional y privada; puntos de vista del personaje, cambios de actitud del personaje, personalidad o tono y cómo se enfrenta a los conflictos, se corresponderían con la pregunta número 1 de Barr (2002). Estas 13 preguntas se incorporan una vez que el alumno ha trabajado en taller $1 \mathrm{con}$ las 8 preguntas más 2. Cuando ya las tiene suficientemente metabolizadas entonces integramos estas 13 en el taller 2, que le ayudarán a profundizar aún más y ofrecen otros puntos de vista a una misma pregunta planteada por Barr (2002).

En cuanto a Información sobre el guion también coincide con la pregunta de Barr (2002): ¿qué más información sobre usted se puede deducir de la historia? La novedad la ofrece su relación con los demás, que es una de las incorporaciones de la docente a las de Barr (2002), qué hace cuando está solo y momento de revelación del personaje. Habitualmente se propone al alumnado trabajar con el actor o proponer que lo entrene por su cuenta, un "momento privado" del personaje en el que hace algo que no haría frente a nadie (Strasberg, 1990). Este acto provoca una intimidad e indagación profunda de la necesidad básica del personaje (Batson, 2014). A su vez compartir con el actor lo que el director cree que aprende el público de la historia y lo que aprende el personaje es un acercamiento a la construcción bastante profundo. Por último, que el director comparta cómo imagina físicamente al personaje ayuda mucho en el proceso.

Una vez terminada la fase analítico-teórica se pasa a la fase empírica, que está compuesta por dos talleres prácticos y el examen final, sustentada en ABP y en learn by doing. Esta fase empírica consta de tres fases: en la fase 2 se da el taller 1, en la fase 3 el taller 2, y en la fase 4 el examen final.

El taller 1 es más directivo por parte del docente quien actúa como coachentrenador. Para este trabajo hay asignada una escena específica que el alumno debe dirigir. Cada discente ejercerá de director, pero también de actor en otra escena de otro 
compañero, para vivir desde dentro bloqueos habituales del actor. El director-alumno hará su trabajo previo de mesa, de análisis a través del protocolo de las preguntas de Barr más 2. Además de las preguntas el alumno-director traerá a clase una idea de dirección que recoja un esquema de movimiento y unas acciones físicas transformadoras según el Sistema de Stanislavski. No ha habido ensayo previo pues se trata de hacer un simulacro de un primer día de ensayo para trabajar el aspecto comunicativo del director con el actor. En este contexto el alumno ha de poner en práctica todas las competencias de comunicación, dirección y liderazgo estudiadas en la fase 1.

Conocer las 8 preguntas de Barr (2002) facilitan QUÉ información da el director al actor. La pregunta sobre si el actor ha vivido una situación similar facilita uno de los CÓMO abordar la interpretación. Si la respuesta del actor es que no lo ha vivido una opción es la imaginación, el "sí mágico", ayudar al actor a imaginar a través de preguntas: "si tú fueras ese personaje, ¿cómo pensarías?, ¿cómo hablarías?, ¿cómo te moverías". Se trata de incitarle a vivir en un como si. En cambio, si el actor sí ha vivido una situación similiar, y siempre que esté preparado, puede sumergirse en la memoria emotiva, evocando un hecho del pasado de iguales características, que se trae al momento presente en forma de transferencia (Hagen, 2002). Para el CÓMO también son necesarios todos los puntos relativos a las claves, reglas, características de dirección y liderazgo mencionadas. Este taller supone un $40 \%$ del porcentaje de Actividad Académica dirigida.

Del taller 1 -básico- se da un salto más a la complejidad con el taller 2 donde la docente pasa a ser un guía. En este caso el alumno ha elegido su propia escena de las ofertadas en el Campus virtual o de su elección, y ha tenido 3 semanas para ensayar con sus actores -previamente elegidos por el alumno entre sus compañeros de clase-. A su vez el director habrá hecho su trabajo previo de análisis con protocolo de 8 preguntas de Barr más 2 (2002), y como novedad se incorporan las 13 preguntas de Serna (1999). Además, hará una planificación de ensayos y una bitácora de dirección con acciones. A todos los puntos trabajados en el taller 1 se suman la búsqueda de beats, tácticas, obstáculos, subtexto, y súper objetivo para hacer un análisis del texto más profundo. El resultado final a modo de ensayo general se muestra en clase. Este taller más complejo supone un $60 \%$ del porcentaje de Actividad Académica dirigida.

La fase final, fuera del contexto de los talleres, es la dirección y grabación de un monólogo con un actor externo que supone el examen final. Este monólogo ha debido ser ensayado y grabado profesionalmente. Este es el único caso en el que se valora el resultado final y la docente está ausente del proceso, aunque está disponible para tutorías. El alumno adjuntará un documento que contiene el protocolo de taller 1 y 2 , además de una bitácora de dirección detallada día a día. Puesto que este examen final supone la culminación de la creatividad y autonomía total del discente, el esfuerzo del alumno irá dirigido no solo a la aplicación de una metodología de dirección, sino a la creación de un estilo propio en su creatividad. El examen final supone un $50 \%$ de la nota global.

\subsection{El rol del docente}

Durante la asignatura el docente adquiere varios roles diferenciados. En la primera fase analítico-descriptiva el profesor tiene el rol de experto en instrucción según la propuesta de pedagogía de la coasoaciación de Prensky (2013). El profesor aportará todo el 
conocimiento posible sobre la materia, pero también toda la imaginación y creatividad posible para que al alumno le resulte atractivo y efectivo.

En los talleres prácticos, en la fase 2, taller 1, el profesor es entrenador y en la fase 3, taller 2, es guía. En estas fases no existe la teoría, solo la acción. El taller 1 al simular un primer ensayo, el maestro ilustra en algún caso, cuando tras observar percibe que el alumno lo necesita. Esto les motiva y acompaña. Para el taller 2 con ensayos fuera del aula y un último ensayo general en el aula, el alumno ya tiene experiencia y un protocolo de trabajo, lo que le permite ser más autónomo y no necesitar del profesor. Por ello el docente-guía será un mero ayudante del alumno motivado (Prensky, 2011).

\subsection{La rúbrica de la creatividad}

La creatividad, entendida como la capacidad de crear, es una competencia clave de la asignatura. A esta definición básica de la RAE le sumamos los componentes de novedad que parecen tener en común todas las definiciones, a pesar de que "no existe una definición unitaria de creatividad" (Matussek, 1984, p.11).

Para Hernández (1999) se trata de un:

conjunto de aptitudes vinculadas a la personalidad del ser humano que le permiten, a partir de una información previa, y mediante una serie de procesos internos (cognitivos), en los cuales se transforma dicha información, la solución de problemas con originalidad y eficacia (p.67).

A continuación, mostramos de la rúbrica la parte relativa a la creatividad. Recordemos que esta necesita de dos tipos de pensamiento: el lógico o lineal, que nos ayuda con el proceso lógico; y el pensamiento lateral, que desorganiza la secuencia lógica para llegar a soluciones partiendo de otro ángulo. Los dos son importantes. Con el segundo generamos las ideas y con el primero las desarrollamos y utilizamos (De Bono, 1998) (Tabla 3).

Tabla 3.

La rúbrica de la creatividad.

\begin{tabular}{|c|c|c|c|}
\hline TALLER 1 & $\begin{array}{l}\text { Poco o nada } \\
\text { competente }\end{array}$ & Bastante competente & Muy competente \\
\hline & $\begin{array}{l}\text { No emplea el } \\
\text { pensamiento lateral en la } \\
\text { propuesta de dirección de } \\
\text { la escena trabajada. } \\
\text { Únicamente se guía por la } \\
\text { lógica y contesta al } \\
\text { protocolo de preguntas de } \\
\text { Tony Barr. Cuando el } \\
\text { docente-entrenador } \\
\text { propone un cambio no } \\
\text { sabe gestionarlo. }\end{array}$ & $\begin{array}{l}\text { Emplea pensamiento } \\
\text { lateral y lineal en } \\
\text { muchos momentos y } \\
\text { ofrece una visión propia } \\
\text { y original en la dirección } \\
\text { de la escena. } \\
\text { Cuando el docente- } \\
\text { entrenador propone un } \\
\text { cambio el alumno lo } \\
\text { aborda eficazmente. }\end{array}$ & $\begin{array}{l}\text { En todo momento es } \\
\text { habilidoso en el uso } \\
\text { conjunto } \\
\text { pensamiento lateral y } \\
\text { lineal y ofrece una visión } \\
\text { propia y original } \\
\text { excelente en la dirección } \\
\text { de la escena, tanto en } \\
\text { los materiales escritos } \\
\text { como en la puesta. } \\
\text { Cuando el docente- } \\
\text { entrenador propone un } \\
\text { cambio el alumno lo } \\
\text { aborda eficaz y } \\
\text { excelentemente. }\end{array}$ \\
\hline
\end{tabular}


Tabla 3.

La rúbrica de la creatividad. (Continuación).

\begin{tabular}{|c|c|c|c|}
\hline \multirow[t]{2}{*}{ TALLER 2} & Poco o nada competente & Bastante competente & Muy competente \\
\hline & $\begin{array}{l}\text { No emplea el } \\
\text { pensamiento lateral en la } \\
\text { propuesta de dirección } \\
\text { tanto en los ensayos como } \\
\text { en la elaboración de los } \\
\text { materiales de trabajo y el } \\
\text { ensayo con público en } \\
\text { clase. Únicamente se guía } \\
\text { por la lógica y contesta al } \\
\text { protocolo de preguntas de } \\
\text { Tony Bar, 13 preguntas } \\
\text { del actor al director, pero } \\
\text { no las relaciona entre sí. } \\
\text { Su propuesta de dirección } \\
\text { no es original. } \\
\text { Cuando el docente-guía } \\
\text { propone un cambio el } \\
\text { alumno no sabe } \\
\text { gestionarlo. }\end{array}$ & $\begin{array}{l}\text { Emplea pensamiento } \\
\text { lateral y lineal en muchos } \\
\text { momentos y ofrece una } \\
\text { visión propia y original en } \\
\text { la dirección de la escena } \\
\text { tanto en los ensayos como } \\
\text { en la elaboración de los } \\
\text { materiales de trabajo y el } \\
\text { ensayo con público en } \\
\text { clase. } \\
\text { Cuando el docente- } \\
\text { entrenador propone un } \\
\text { cambio el alumno lo } \\
\text { aborda eficazmente. }\end{array}$ & $\begin{array}{l}\text { En todo momento es } \\
\text { habilidoso en el uso } \\
\text { conjunto de } \\
\text { pensamiento lateral y } \\
\text { lineal y ofrece una } \\
\text { visión propia y original } \\
\text { excelente en la } \\
\text { dirección de la escena, } \\
\text { tanto en los materiales } \\
\text { escritos como en la } \\
\text { puesta. } \\
\text { Cuando el docente- } \\
\text { entrenador propone un } \\
\text { cambio el alumno lo } \\
\text { aborda } \\
\text { excelentemente. }\end{array}$ \\
\hline $\begin{array}{l}\text { EXAMEN } \\
\text { FINAL }\end{array}$ & $\begin{array}{l}\text { No emplea el } \\
\text { pensamiento lateral en la } \\
\text { propuesta de dirección } \\
\text { tanto en los ensayos como } \\
\text { en la elaboración de los } \\
\text { materiales de trabajo y en } \\
\text { la grabación del } \\
\text { monólogo. Únicamente se } \\
\text { guía por la lógica y } \\
\text { contesta al protocolo de } \\
\text { preguntas de Tony Bar, 13 } \\
\text { preguntas del actor al } \\
\text { director y menciona } \\
\text { alguna harramienta } \\
\text { actoral usada pero no } \\
\text { profundiza en ella. }\end{array}$ & $\begin{array}{l}\text { Emplea pensamiento } \\
\text { lateral y lineal en muchos } \\
\text { momentos y ofrece una } \\
\text { visión propia y original en } \\
\text { la dirección de la escena } \\
\text { tanto en los ensayos como } \\
\text { en la elaboración de los } \\
\text { materiales de trabajo y en } \\
\text { la grabación del } \\
\text { monólogo. } \\
\text { Contesta al protocolo de } \\
\text { preguntas de Tony Bar, } 13 \\
\text { preguntas del actor al } \\
\text { director y menciona más } \\
\text { del } 50 \% \text { de las } \\
\text { herramientas actorales } \\
\text { vistas en la fase } 1 \text { y las } \\
\text { emplea eficazmente. }\end{array}$ & $\begin{array}{l}\text { En todo momento es } \\
\text { habilidoso en el uso } \\
\text { conjunto de } \\
\text { pensamiento lateral y } \\
\text { lineal y ofrece una } \\
\text { visión propia y original } \\
\text { excelente en la } \\
\text { dirección de la escena, } \\
\text { tanto en los materiales } \\
\text { escritos como en la } \\
\text { puesta y en la } \\
\text { grabación. } \\
\text { Contesta al protocolo } \\
\text { de preguntas de Tony } \\
\text { Bar, 13 preguntas del } \\
\text { actor al director y } \\
\text { menciona el 100\% de } \\
\text { las herramientas } \\
\text { actorales vistas en la } \\
\text { fase } 1 \text { y las emplea } \\
\text { eficaz y } \\
\text { excelentemente y las } \\
\text { relaciona entre sí. }\end{array}$ \\
\hline
\end{tabular}

Fuente: elaboración propia 


\section{RESULTADOS}

A continuación, se muestran algunos ejemplos significativos de Grado y Máster, que constatan la asimilación y aumento de la creatividad y autonomía en el alumnado de modo gradual desde el taller 1 y taller 2 hasta el examen final.

\subsection{Actividad dirigida-taller 1}

En el año 2013-2014 la alumna A.G. comentaba sobre su trabajo de dirección de una escena de la película Días de fútbol para la actividad dirigida-taller 1: "el primer taller de dirección escénica ha sido un primer acercamiento práctico a todas las reglas y pautas aprendidas en las clases de teoría. A lo largo del trabajo la alumna explica en detalle las 8 preguntas de Barr más 2, y comenta: "con este primer acercamiento a la escena y al guion y las primeras relaciones actor-director donde se aclaran dudas y se transmite información finalizamos satisfactoriamente el taller 1" (González et al., 2020, p.2).

Sobre el análisis de escena y construcción de personaje en el taller 1 la alumna N.L. comenta: "el análisis de los personajes me ayudó a saber qué quería en la escena, cuál era el entorno y sobre todo los objetivos que impulsaban a los personajes en la acción dramática. Mi objetivo como directora era ayudar a los actores a entender a los personajes como uno que actúa de forma progresiva, que escucha realmente en escena y cuyas emociones varían en la relación con el resto de personajes. Una de las principales cosas que he aprendido en relación a este trabajo de mesa es que, por mucho que pienses que todo está claro, es muy bueno e importante que el actor pregunte y acerque el personaje a lo que él piensa que puede ser. De este modo, el director puede contrastar e ir puliendo hasta que consigue lo que quiere" (González et al., 2020, pp.13-14).

\subsection{Actividad dirigida-taller 2}

Esta misma alumna (N.L.) durante el taller 2 explica: "el trabajo con mis dificultades me demostró que éste es un aprendizaje constante y que lo importante no está sólo en el resultado, sino que también lo está en el proceso".

La alumna A.G. refleja la importancia de la gradación entre un taller y otro. "Este taller 2 irá evolucionando con la información añadida y complementada que se recoge de manera rigurosa en el taller 1. Ensayar de forma continuada, trabajando meticulosamente en cada aspecto del guion, ayudará a conseguir con mayor probabilidad el triunfo de la interpretación. Lo primero que debe hacer el director es informar, el guion no proporciona toda la información necesaria, ya que, a menudo carece de descripciones psicológicas del personaje. Gracias a la información que se da a los actores de sus personajes, el director aporta seguridad en ellos. Así mismo, se debe comentar la interpretación, reforzando primero lo positivo, usar un buen lenguaje, no pretender que los actores imiten tu forma de actuar, y no marcar acciones externas para reflejar sentimientos. Por último, he utilizado para los ensayos otras técnicas de Stanivsllavski el "sí mágico" aportando la máxima realidad a las acciones. Así mismo, he usado la técnica de la "memoria emotiva" intentando que los actores recordaran sus reuniones más graciosas con amigos y trajeran los recuerdos a la escena" (González et al., 2020, p.3). 
Los comentarios de A.B. ilustran el aumento de creatividad en cuanto a capacidad creadora y el incremento de autonomía en su dirección de una escena de la película Te doy mis ojos. "Otra de las claves para dirigir que mantuve en todo momento fue "negociar" con las actrices y no imponer. Permití en todo momento como directora intérprete o pedagoga (Nemiróvich-Dánchenko, 2006), que actuaran con sus propios recursos, elementos analíticos e impulsos creativos para así poder sentir los procesos internos del papel en todos sus matices sabiendo ponerme en el lugar de las actrices, sin olvidar su individualidad y apreciando y desarrollando sus facultades creativas. Además, como directora espejo (Nemiróvich-Dánchenko, 2006) supe reflejar sus cualidades individuales y empaticé con ellas desde un primer momento. Escuchaba sus recursos, los cuales me servían para la dirección que les proponía para la escena. (...) Algo que les ayudó fue lo que llamaba Stanivslaski, circunstancias dadas, aquellas circunstancias que ayudan a saber de dónde viene y hacia dónde va. (...) En la primera lectura de guion indagué en la relación entre el texto y el subtexto. Al análisis literario, que explica qué dicen y qué hacen los personajes, sumé el análisis dramático que contesta a por qué hablan y por qué actúan los personajes. Como en toda escena, es imprescindible buscar un conflicto. Establecí el conflicto principal que se desarrolla a lo largo de la línea de sucesos, partiendo de una acción que buscaba la madre pero que no la continuaba su hija. (...) En cuanto a la construcción del personaje utilicé la técnica del "si condicional" o "sí mágico" que consiste en poner al actor en el supuesto "si yo fuera...", como rápida identificación con el problema del personaje", explicó. La alumna también hizo uso en ocasiones de la memoria emotiva, "técnica introspectiva a través de la cual se le propone al actor recuperar, recordar, evocar, y hacer presentes experiencias emotivas de su propia vida para utilizarlas en el proceso de creación de su personaje" comentaba A.B. En relación a la construcción del personaje la alumnadirectora permitió a "las actrices aportar al papel las partes de sí mismas que eran adecuadas para la interpretación de los personajes, trabajar a partir de sí mismas en todo momento, y dejando a un lado partes de sí mismas que no son adecuadas para el papel" (González et al., 2020, pp. 20-25). En cuanto a las "reglas para dirigir actores" la alumna favoreció la atmósfera para que crecieran las emociones. En este taller Ballesteros también implementó el método de las acciones física de Stanivslaki -MAF-, con un por qué y un para qué.

El año 2019-20 los alumnos del Máster de series de ficción, D.P. y A.P., tras los análisis de los protocolos de preguntas, se daban cuenta de que "la escena era compleja en todas sus dimensiones, por lo que decidimos incluir un análisis DAFO para percatarnos de la verdadera naturaleza de la escena a estudiar y ser conscientes de nuestras responsabilidades como directores", explican. "Finalmente concluimos los ensayos de forma placentera al lograr conjuntamente un trabajo sorprendente, con un resultado honesto y verdadero gracias a los ensayos, que fueron preparados de forma exhaustiva según nuestro análisis DAFO. Estamos orgullosos de haber formado parte de este viaje hacia la verdad al llevar a cabo esta escena a su realidad interpretativa" (González et al., 2020, pp. 36-37).

\subsection{Examen final-monólogo}

De todas las propuestas de trabajo, en Grado, la dirección y grabación del monólogo examen final- es el culmen de la autonomía del alumno-director, y de su capacidad máxima de creación, pues no hay ningún tipo de intervención ni feedback por parte del docente hasta finalizar el proceso.

El caso de L.P. revela cómo algunos directores ya en su trabajo de mesa tienen revelaciones sobre el personaje. En su trabajo de un monólogo del film Mónster comenta: "la mayor clave, y cuando logramos los resultados que yo quería, vinieron tras la indicación que describo en la pregunta 3 de las 13 preguntas" (González et al., 2020, 
p.50), lo que le llevó a trabajar la personalización con la actriz quien debía imaginar frente a sí a tres personajes diferentes.

La propuesta de dirección del monólogo de la película Psicosis por A.E. el año 2015-2016 refleja el grado de autonomía y creatividad que se logra tras pasar por los talleres. "Enfrentarme a este trabajo no ha sido nada fácil. Como trabajo final de asignatura quería arriesgarme. No quería lo fácil y cómodo. Quería conocer hasta donde podía llegar" (González et al., 2020, p.27), concluye A.E.

\section{CONCLUSIONES}

La enseñanza de la asignatura "Dirección de actores", percibida por los alumnos como "subjetiva" por su carácter artístico, se "objetiviza" con una sistematización de contenidos y protocolos.

En ese sentido, la estructuración y sistematización de pautas y esquemas de trabajo son la base de la creatividad. A su vez, una base teórico-práctica de metodologías y herramientas interpretativas orienta la dirección de actores, así como un marco teórico bien definido le permite luego pasar a la práctica.

Dicho marco teórico es necesario como base para la creatividad y autonomía.

En la parte empírica de la asignatura los talleres necesitan un protocolo de análisis y construcción de personajes que se materializan en las 8 preguntas de Tony Barr más 2, y las 13 preguntas de Serna. También en la parte empírica se entrenan las herramientas interpretativas básicas y competencias genéricas de dirección de la fase descriptiva. En estos casos, cuánto más detalladas son las respuestas de las preguntas de Barr y Serna, mayor información tienen los actores y mayor creatividad, verdad y matices ofrecerá su interpretación.

También cuánto más específica es la explicación del vínculo entre personajes y su relación con el entorno, más creíble es la actuación.

La creatividad del actor es proporcional a la información que le da el director.

El $90 \%$ de la información que se necesita está en el trasfondo de la escena u objetivo (Barr, 2002).

El sí mágico y la memoria emotiva de Stanislavski son dos herramientas que sumergen al actor en la verdad del personaje.

Es óptimo que exista una gradación en la adquisición de las herramientas y su puesta en práctica. De hecho, el éxito de la asignatura reside en su sistematización y su evolución por fases y gradual. Del taller 1 al 2 el alumno gana autonomía y esto le permite aumentar su creatividad. Del taller 2 al monólogo-examen final se da el mayor salto en independencia y creación.

En definitiva, la metodología de ABP y learn by doing son las más adecuadas para los talleres prácticos de la asignatura.

Los talleres basados en ABP y learn by doing suponen la culminación de adquisición de competencias por parte del alumnado, su entrenamiento y corroboración del método de la asignatura "Dirección de actores".

La única forma de conseguir que los estudiantes aprendan de un modo independiente y autónomo es enfrentándoles a situaciones en las que tienen que utilizar estrategias de búsqueda de información, tomar decisiones y aplicar los conocimientos adquiridos a la solución de problemas realistas (De Miguel, 2009). 


\section{REFERENCIAS}

Adler, S. (2000). The art of acting. Massachusetts: Applause.

Barron, F., \& Harrington, D., (1981) Creativity, Intelligence, and Personality. Annual Review of Psychology, 32(1), 439- 476.

Barr, T. (2002). Actuando para la cámara. Manual de actores para cine y TV. Madrid: Plot.

Barrows, H.S. (1986). A Taxonomy of problem-based learning methods. Medical Education, 20 (6), 481-486. https://doi.org/10.1111/j.1365-2923.1986.tb01386.x

Batson, S. (2014). Truth, Personas, Needs, and Flaws in the Art of Building Actors and Creating Characters. New York: Webster/Stone.

Benito, A., \& Cruz, A. (2005). Nuevas claves para la docencia universitaria en el Espacio Europeo de Educación Superior. Madrid: Narcea.

Boleslavsky, R. (1954). La formación del actor: las seis primeras lecciones, México: Editorial Alameda.

Bogart, A., \& Vieites, M. F. (2006). Introducción a Dirección de Escena. Santiago de Compostela: Galaxia.

Caine, M. (2003). Actuando para el cine. Madrid: Plot.

Canfield, C. (2004). El arte de la dirección escénica. Madrid: ADE.

Chejov, M., (1999). Sobre la técnica de la actuación. Barcelona: Alba editorial.

De Bono, E. (1994) El pensamiento creativo. Madrid: Paidós Ibérica.

De Miguel, M. (2009) (coord.). Metodologías de enseñanza para el desarrollo de competencias. Orientaciones para el profesorado universitario ante el Espacio Europeo de Educación Superior. Madrid: Alianza.

De Miguel, R. (2005). La observación sistemática y participante como herramienta de análisis de los fenómenos comunicativos. En M.A. Berganza \& J.A. Ruiz, Investigar en comunicación (277-293). Madrid: McGraw-Hill.

Eines, J. (2007). La formación del actor. Barcelona: Gedisa editorial.

González, A. et al. (2020). Datos de investigación resultados alumnos Dirección de Actores. https://bit.ly/3oL6khN

Grotowski, J. (2009). Hacia un teatro pobre. Madrid: Siglo XXI.

Hagen, U. (2002). Un reto para el actor. Madrid: Alba Editorial.

Layton, W. (2000). ¿ Por qué? Trampolín del actor. Madrid: Fundamentos.

Nemiróvich-Dánchenko, V. (2006). En la senda de Stanislavski. Madrid: Fundamentos.

Meisner, S. (2003). Sobre la actuación. Madrid: La Avispa.

Melendres, J. (2000). La direcció dels actors. Diccionari mínim. Barcelona: Institut del Teatre de la Diputació de Barcelona.

Meyerhold, V.E. (2010). Lecciones de dirección escénica (1918-1919). Madrid: Asociación de Directores de Escena.

Miralles, A. (2010). La dirección de actores en cine. Madrid: Cátedra.

Morales, C. (2017). La creatividad, una revisión científica. Arquitectura y Urbanismo, XXXVIII (2), 53-62. Ciudad de La Habana: Instituto Superior Politécnico José Antonio Echeverría.

Prensky, M. (2013). Enseñar a nativos digitales. México: SM Ediciones.

Serna, A. (1999). El trabajo del actor de cine. Madrid: Cátedra.

Stanislavski, K. (2003). El trabajo del actor sobre sí mismo en el proceso creador de la vivencia. Barcelona: Alba.

Stanislavski, K. (2002). La construcción del personaje. Madrid: Alianza Editorial.

Sternberg, R.J. (1999). Handbook of creativity. Cambridge: University Press

Strasberg, L. (1990). Un sueño de pasión: la elaboración del método. Buenos Aires: Icaria.

Weston, J. (2004). La dirección de actores, cómo obtener actuaciones memorables en cine y televisión. La Coruña: Fluir Ediciones. 